\title{
Nachhaltige Digitalisierung in Unternehmen
}

Digitalisierung und Nachhaltigkeit gehören nicht nur in der Wissenschaft, sondern auch im Alltagsleben zu den großen Megatrends der Zukunft. Häufig werden diese beiden Themen jedoch nur isoliert innerhalb einer Fachdisziplin und nicht im Zusammenhang betrachtet, obwohl gerade deren Vernetzung interessante Lösungen für aktuelle Herausforderungen liefert.

Michael Jacob 
Digitalisierung wird durch den Einsatz von Informationstechnik erreicht und führt häufig zu einer Automatisierung von bisher manuellen Tätigkeiten. In der Summe entsteht dadurch ein digitaler Wandel, der die Gesellschaften derzeitig stark verändert. Aktuelle Entwicklungen im Bereich der Digitalisierung lassen sich mit folgenden Schlagworten beschreiben: künstliche Intelligenz, Big Data, Augmented Reality, Internet der Dinge, Cloud Computing und Blockchain.

Das Thema Nachhaltigkeit ist durch den Klimawandel und andere umweltbezogene Herausforderungen in den Fokus der Öffentlichkeit gelangt. Nachhaltigkeit bedeutet jedoch mehr als die Lösung ökologischer Probleme. Vielmehr gilt es, wie aus der Abb. 1 ersichtlich, ökologische, ökonomische und soziale Aspekte in Einklang miteinander zu bringen. Die Theorie stellt diese Dimensionen und deren Zusammenhänge in sogenannten Nachhaltigkeitsmodellen dar. Bekannt sind hier insbesondere das einfache und gewichtete Dreisäulenmodell, das Nachhaltigkeitsdreieck und das Schnittstellenmodell. Eine Detaillierung zu diesen klassischen Modellen bilden die "Zauberscheiben der Nachhaltigkeit“.

Ergänzend zu diesen Nachhaltigkeitsmodellen, entwickelten sich in der Literatur Nachhaltigkeitsstrategien. Während das Prinzip der Effizienz eine ergiebigere Nutzung von Materialien und Energie anstrebt, versucht die Suffizienz den Res-

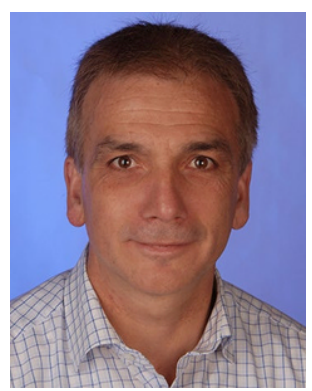

Prof. Dr. Michael Jacob ( $\)$

ist Professor für Betriebswirtschaft an der Hochschule Kaiserslautern und wissenschaftlicher Leiter am Institut für Betriebswirtschaft und Wirtschaftsinformatik.

$\mathrm{Zu}$ dem Themenbereich hat der Autor bei Springer Vieweg das Buch „Digitalisierung und Nachhaltigkeit" veröffentlicht. michael.jacob@hs-kl.de

Hochschule Kaiserslautern Amerikastr. 1, 66482 Zweibrücken, Deutschland

\section{Abb. 1 Ziele und Strategien einer nachhaltigen Digitalisierung}

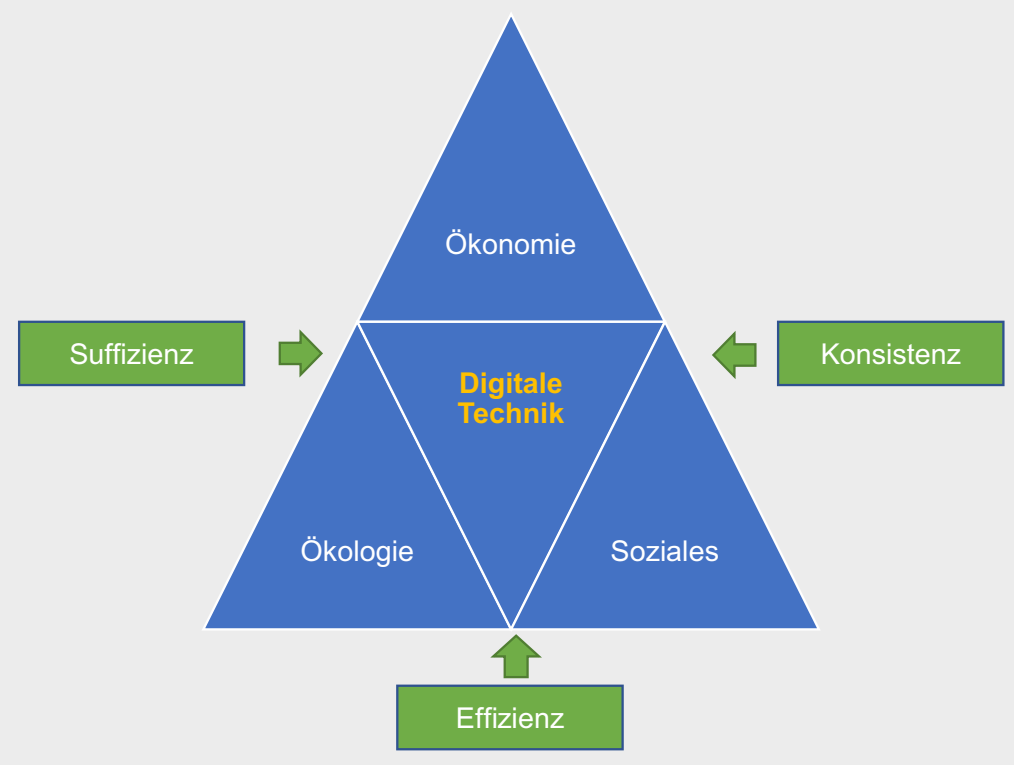




\section{Zusammenfassung}

- Digitalisierung wird aktuell insbesondere durch künstliche Intelligenz, Big Data, Augmented Reality, Internet der Dinge, Cloud Computing und Blockchain vorangetrieben.

- Nachhaltigkeit lässt sich durch Effizienz, Suffizienz und Konsistenz erreichen.

- Nachhaltige Digitalisierung verändert betriebliche Grundstrukturen sowie Leistungs-, Unterstützungsund Managementprozesse.

sourcenverbrauch zu reduzieren und die Konsistenz Technik und Natur miteinander in Einklang zu bringen. Nachteilig wirken sich in diesem Zusammenhang Reboundeffekte aus. So kann zum Beispiel ein geringerer Kraftstoffverbrauch von Kraftfahrzeugen bei konstanten oder sinkenden Preisen zu einer Zunahme der gefahrenen Kilometer führen, was den zuvor erzielten Einspareffekt dann kompensiert oder sogar überkompensiert.

\section{Grundsatzfragen und betriebliche Grund- strukturen}

Insbesondere für Unternehmen bietet es sich an, die Digitalisierung nicht zu maximieren, sondern ökologisch, ökonomisch und sozial verantwortlich zu gestalten. Hierbei ist $\mathrm{zu}$ beachten, dass die entscheidungstheoretischen Zielbeziehungen nicht nur zwischen der Digitalisierung und Nachhaltigkeit, sondern auch zwischen den einzelnen Dimensionen der Nachhaltigkeit komplementär, konkurrierend oder neutral sein können. Häufig existieren Zielkonflikte, die von der obersten Unternehmensführung im Rahmen der Corporate Social Responsibility zu entscheiden sind. Insbesondere gilt es zu verhindern, dass "Greenwashing“ oder „Bluewashing“ betrieben werden, die als Folge der durch die Digitalisierung entstandenen Transparenz zu sogenannten "Shitstorms“ in den sozialen Medien führen können.

Von besonderer Bedeutung bei der integrierten Betrachtung der Digitalisierung und der Nachhaltigkeit sind die Geschäftsmodelle von Unternehmen. Als neues Modell hat sich hier in den letzten Jahren die "Sharing Economy“ entwickelt. Unternehmen wie Uber oder Airbnb ist es gelungen, mithilfe von Internetplattformen ganze Branchen disruptiv zu verändern. Nicht immer sind hiermit jedoch positive Auswirkungen im Bereich der Nachhaltigkeit verbunden, da zum
Beispiel negative soziale Effekte am Wohnungsmarkt oder im Transportgewerbe zu beobachten sind.

Auch die Kreislaufwirtschaft versucht, Digitalisierung und Nachhaltigkeit sinnvoll miteinander zu verknüpfen. Während sie sich im engeren Sinne nur auf die Abfallwirtschaft bzw. das Recycling bezieht, umfasst die erweiterte Form der Kreislaufwirtschaft auch die Produktentwicklung und die Produktnutzung. Die digitale Kennzeichnung von Produktbestandteilen führt hier zu sehr viel weiter gehenden Möglichkeiten in Bezug auf die Wiederverwertbarkeit.

Teilweise widersprüchlich wird die Digitalisierung von Produkten unter Nachhaltigkeitsaspekten diskutiert. Digitale Produkte wie zum Beispiel ein Musikstream oder ein E-Book sind ökologisch näher betrachtet häufiger weniger umweltfreundlich als ihr analoges Pendant. Zu berücksichtigen ist bei diesem Kalkül insbesondere die Produktion der digitalen Geräte, deren Stromverbrauch, deren Nutzungsintensität und deren Entsorgung, die oft unter fragwürdigen Umständen in Entwicklungsländern stattfindet. Ebenso problematisch ist insbesondere in Verbindung mit digitalen Produkten die $\mathrm{Zu}$ nahme der Wegwerfmentalität von Verbrauchern. Ob Unternehmen diese mit einer geplanten technischen Obsoleszenz fördern, ist nicht einfach zu beweisen. Jedoch ist allgemein festzustellen, dass Produktlebenszyklen immer kürzer werden und defekte Teile nur schwer austauschbar sind. Verstärkend kommt die psychologische Obsoleszenz hinzu, die dazu führt, dass unter Prestigeaspekten zum Beispiel ein Austausch des Smartphones vorgenommen wird, obwohl dies technisch nicht notwendig wäre.

Auch der Themenkomplex „Green IT“ verbindet die Digitalisierung mit der Nachhaltigkeit. Kernthema ist hier die Frage, wie der Ressourcen- und Energieverbrauch von Rechenzentren und Informationstechnik gesenkt werden kann. Dies ist umso wichtiger, da Schätzungen davon ausgehen, dass weltweit der Betrieb von Informationstechnik genauso viel Kohlendioxid verursacht wie der Flugverkehr. Fortschritte in der Technik sind in den letzten Jahren zwar entstanden, jedoch stellt sich die Frage, ob die längere Nutzung der „alten“ Technik insgesamt betrachtet nicht nachhaltiger wäre.

Auch das richtige Datenmanagement kann neben nachhaltiger Hard- und Software zu mehr Nachhaltigkeit führen. Beispielsweise können Informationen zum Bedarf von Produktion zur Vermeidung von Überproduktion führen. $\mathrm{Zu}$ vermeiden sind jedoch Datensammlungen ohne nachhaltigen Zweck, was prinzipiell durch „Big Data“ möglich ist. In 
diesem Zusammenhang sind auch der Datenschutz und die Corporate Digital Responsibility von besonderer Bedeutung.

$\mathrm{Ob}$ neuere Entwicklungen im Bereich der Digitalisierung, wie zum Beispiel Cloud-Computing, künstliche Intelligenz, Blockchain, das Internet der Dinge oder Augmented Reality zu mehr Nachhaltigkeit führen, lässt sich nicht eindeutig beantworten, da von diesen Techniken grundsätzlich positive und negative Wirkungen in Bezug auf die Nachhaltigkeit ausgehen. So kommen Studien beispielsweise zu dem Ergebnis, dass künstliche Intelligenz zu einer Erreichung der Nachhaltigkeitsziele der Vereinten Nationen führen kann. Jedoch gibt es ebenso Potenziale der künstlichen Intelligenz, die bestehende Ungleichheit verschärfen und damit zu weniger Nachhaltigkeit führen. Die Blockchain-Technologie ist in Verbindung mit dem Bitcoin sehr populär geworden. Kritisiert wurde in Bezug auf den Energieverbrauch, dass dieser je nach Modellrechnung mit dem Energieverbrauch eines Landes wie Nigeria vergleichbar wäre. Positiv ist dagegen die Möglichkeit, mithilfe der Blockchain-Technologie die Herkunft von Lebensmitteln zurückverfolgen zu können.

\section{Leistungsprozesse}

Neben dem Einfluss von Digitalisierung und Nachhaltigkeit auf unternehmerische Strukturen gilt es ergänzend, deren Einfluss auf betriebliche Prozesse zu evaluieren. Diesbezüglich sind zunächst Prozesse der Material- und Produktionswirtschaft relevant. Unternehmen werden hier auf Druck der Öffentlichkeit immer mehr gezwungen, Verantwortung für ihre Wertschöpfungskette zu übernehmen. Die Wertschöpfungskette beginnt meistens mit den Lieferanten, die wiederum Vorlieferanten haben können. Um soziale oder ökologische Problembereiche herausfiltern zu können, existieren vielfältige Softwarelösungen, die es erlauben, Nachweise von den Lieferanten einzufordern und diese auch zu auditieren.

Von besonderer Relevanz ist auch das Thema „Green Logistics", das untersucht, wie sich negative Umweltauswirkungen von Verkehrsträgern reduzieren lassen. Da der Logistiksektor für einen Großteil des Kohlendioxidausstoßes verantwortlich ist, sind digitale Lösungen zur Reduzierung des Problems von besonderer Bedeutung. Mit einem Statistikmodul lassen sich beispielsweise Ausreißer beim Benzinverbrauch und beim Fahrverhalten leicht nachvollziehen. Auch kann der vermehrte Einsatz der Radio-Frequency-Identification(RFID)-Technik zu mehr Nachhaltigkeit beitragen. Mithilfe dieses Systems können ganze Warenlieferungen kontaktlos erfasst werden, was zunächst wirtschaftliche Vorteile gegenüber dem klassi- schen Barcode bietet. Gleichzeitig wird damit die Rückverfolgbarkeit von Produkten einfach ermöglicht.

Auch digitale Energiemanagementsysteme können zu einer größeren Nachhaltigkeit, nicht nur für Unternehmen, führen. Aufgrund der Zunahme der erneuerbaren Energien entwickelt sich das bisher zentrale Netz immer mehr zu einem dezentralen Netz. Intelligente Energiemanagementsysteme müssen deshalb die Energie effizient zwischen Produzenten und Verbrauchern aufteilen. Für Verbraucher gilt es, insbesondere die Energie dann zu nutzen, wenn sie kostengünstig zur Verfügung steht.

Der sich häufig nahtlos an den Beschaffungsprozess anschließende Produktionsprozess lässt sich durch Digitalisierung ebenfalls nachhaltiger gestalten. Teilkomponenten sind insbesondere das Instandhaltungs-, das Qualitäts- und das Schadstoffmanagement. Unter dem Begriff „predictive maintenance" erfolgt zum Beispiel mithilfe von Big Data und dem Internet der Dinge eine vorausschauende Wartung von Maschinen, sodass der Produktionsprozess möglichst nicht durch Wartungsarbeiten unterbrochen wird.

Auch der Bereich Marketing und Vertrieb weist neben der Material- und Produktionswirtschaft vielfältige Anwendungsfälle für eine nachhaltige Digitalisierung auf. Zunächst ist festzustellen, dass die Konsumenten als primäre Adressaten dieses Funktionsbereiches bisher überwiegend nicht nachhaltig agieren. Häufig existiert nur eine Scheinnachhaltigkeit, weil damit soziales Prestige verbunden ist. So wird zum Beispiel ein Elektrofahrrad gekauft, kaum genutzt und trotzdem unverändert mit dem Auto gefahren und im Flugzeug geflogen. Um einen tatsächlichen Wandel der Konsumgewohnheiten zu erzwingen, müsste ein radikaler Kulturwandel weg vom derzeit vorherrschenden Konsumismus stattfinden, der bisher jedoch nicht absehbar ist.

Unternehmen können in einzelnen Bereichen diesen Kulturwandel unterstützten. Letztlich würde dies jedoch häufig

\section{Kernthesen}

- Ökologische und soziale Investitionen führen langfristig zu mehr Wirtschaftlichkeit.

- Nachhaltige Digitalisierung kann einen Beitrag zur Lösung des Klimawandels und anderer Umweltprobleme leisten.

- Kulturwandel zu mehr Nachhaltigkeit muss technischen Fortschritt unterstützen. 
$\mathrm{zu}$ einem freiwilligen Verzicht auf Gewinne zugunsten ökologischer und sozialer Zielsetzungen führen, den sich nicht alle Unternehmen leisten können oder leisten wollen. Denkbar wäre zum Beispiel, auf Marketingmaßnahmen insbesondere im Onlinebereich generell zu verzichten oder diese zumindest stark einzuschränken. Alternativ wäre ein (Online-) Marketing denkbar, das bewusst nur informiert und nicht manipuliert. Bestimmte Konsumentengruppen könnten damit gegebenenfalls von Wettbewerbern abgeworben werden, da sie ein ehrliches Marketing zu schätzen wissen.

Neben dem (digitalen) Marketing lässt sich auch der Onlinehandel unter Nachhaltigkeitsaspekten kritisch beleuchten. Die Vision, dass sich der Verkehr auf den Straßen durch die Zunahme des Onlinehandels reduziert, kann bisher noch nicht nachgewiesen werden. Theoretisch ist zwar vorstellbar, dass ein Lkw, der viele Haushalte beliefert, ökologisch günstiger ist als die Fahrt von vielen einzelnen Konsumenten zu unterschiedlichen stationären Geschäften. In der Praxis finden jedoch kaum weniger Fahrten statt, da nicht alle Einkäufe online erledigt werden und die gegebenenfalls gewonnene Zeit durch Fahrten in Bezug zu Freizeitaktivitäten substituiert wird.

\section{Managementprozesse und Unterstützungs- prozesse}

Neben betrieblichen Leistungsprozessen können auch Management- und Unterstützungsprozesse im Sinne einer nach- haltigen Digitalisierung gestaltet werden. Beispielhaft wird auf den Personalbereich und das Nachhaltigkeitscontrolling eingegangen.

Personalabteilungen von Unternehmen stehen vor der Herausforderung, dass sich die Arbeitswelt in den nächsten Jahren mit zunehmender Geschwindigkeit verändert. Diese Veränderungen sind primär durch die Technik induziert. Viele Prognosen gehen derzeit davon aus, dass in bestimmten Branchen wie zum Beispiel in der verarbeitenden Industrie viele Arbeitsplätze durch digitale Technik substituiert werden. Dem halten Optimisten entgegen, dass neue Arbeitsplätze entstehen. Ob diese jedoch Ersatzarbeitsplätze für die ausgeschiedenen Mitarbeiter sein können, muss bezweifelt werden, da sie ein anderes Qualifikationsprofil erfordern.

Neben den beschriebenen Veränderungen auf dem Arbeitsmarkt müssen sich Personalabteilungen von Unternehmen und anderen Organisationen jedoch auch mit Veränderungen in den Abläufen beschäftigen, die durch die Digitalisierung hervorgerufen werden. So wird aktuell wieder die Arbeit im Homeoffice verstärkt diskutiert, da durch Fortschritte in der Digitalisierung diese immer einfacher möglich wäre. Sicher können durch mehr Heimarbeit auch ökologische Vorteile und mehr Flexibilität für den Arbeitnehmer erreicht werden. Gleichzeitig müssen jedoch ebenfalls unter dem Gesichtspunkt der Nachhaltigkeit soziale Auswirkungen einer fehlenden physischen Präsenz bedacht werden. Von Bedeu-

\section{Abb. 2 Digitale Technik im Kontext anderer Einflussfaktoren der Nachhaltigkeit}




\section{Handlungsempfehlungen}

- Digitalisierung und Nachhaltigkeit vernetzt betrachten

- Nachhaltige und digitale Geschäftsmodelle kreativ entwickeln und umsetzen

- Aufbau- und Ablauforganisation systematisch in Bezug auf ökonomisches, ökologisches und soziales Potenzial überprüfen

tung bei allen Digitalisierungsbemühungen ist letztlich, dass diese zu den Mitarbeiten passen und von diesen akzeptiert werden. Denn ohne menschliche Akzeptanz lassen sich keine in der Theorie bestehenden Digitalisierungsvorteile erzielen.

Digitalisierung und Nachhaltigkeit erfordern nicht zuletzt auch eine Messung und eine Beurteilung des Erfolgs. Diese Aufgabe steht traditionell dem Controlling zu. Digitalisierung und Nachhaltigkeitscontrolling benötigen jedoch im Vergleich zum klassischen Controlling ergänzende Instrumente. Die Verknüpfung und integrierte Betrachtung der beiden Themenbereiche sollte deshalb für den Controller über den Einsatz von spezieller Software, die Erstellung von Nachhaltigkeitsberichten und die umweltbezogene Beurteilung digitaler Produkte hinaus neue Chancen aufzeigen, sein Unternehmen in die Zukunft zu führen.

\section{Fazit}

Der Digitalisierungsprozess ist weltweit im Gange. Das Tempo zur Umsetzung nimmt zukünftig voraussichtlich noch weiter zu. Gleichzeitig schreitet der Klimawandel, verbunden mit zunehmenden Naturkatastrophen, weiter voran. Die beiden Themen sollten deshalb nicht nur von Unternehmen mit einer stärkeren Vernetzung betrachtet werden. Dann kann die Digitalisierung, wie in diesem Beitrag an verschiedenen Stellen aufgezeigt, sicher einen Beitrag zu mehr Nachhaltigkeit leisten. Gleichzeitig muss jedoch auch, wie aus der Abb. 2 deutlich wird, erkannt werden, dass Technik allein keine ausreichende Lösungsmöglichkeit sein wird. Nachhaltigkeit erfordert vielmehr auch Überlegungen, wie das derzeit weltweite Bevölkerungswachstum gestoppt werden kann und wie das Konsumniveau jedes Menschen auf ein vernünftiges $\mathrm{Ma}$ zurückgeführt werden kann. Unternehmen leisten sicher einen Beitrag zur Lösung dieser großen Herausforderungen. Letztlich bedarf es jedoch auch einer Veränderung der politischrechtlichen Rahmenbedingungen im internationalen Kontext und eines Kulturwandels in der Bevölkerung. Nur dann können Unternehmen auch ihrer Verantwortung in vollem Umfang nachkommen.

Funding. Open Access funding provided by Projekt DEAL.

Open Access Dieser Artikel wird unter der Creative Commons Namensnennung 4.0 International Lizenz veröffentlicht, welche die Nutzung, Vervielfältigung, Bearbeitung, Verbreitung und Wiedergabe in jeglichem Medium und Format erlaubt, sofern Sie den/die ursprünglichen Autor(en) und die Quelle ordnungsgemäß nennen, einen Link zur Creative Commons Lizenz beifügen und angeben, ob Änderungen vorgenommen wurden.

Die in diesem Artikel enthaltenen Bilder und sonstiges Drittmaterial unterliegen ebenfalls der genannten Creative Commons Lizenz, sofern sich aus der Abbildungslegende nichts anderes ergibt. Sofern das betreffende Material nicht unter der genannten Creative Commons Lizenz steht und die betreffende Handlung nicht nach gesetzlichen Vorschriften erlaubt ist, ist für die oben aufgeführten Weiterverwendungen des Materials die Einwilligung des jeweiligen Rechteinhabers einzuholen.

Weitere Details zur Lizenz entnehmen Sie bitte der Lizenzinformation auf http://creativecommons.org/licenses/by/4.0/ deed.de

\section{Weiterführende Literatur}

[1] Jacob, M. (2019). Digitalisierung und Nachhaltigkeit. passim 